\title{
Medical aid system: Change way overdue
}

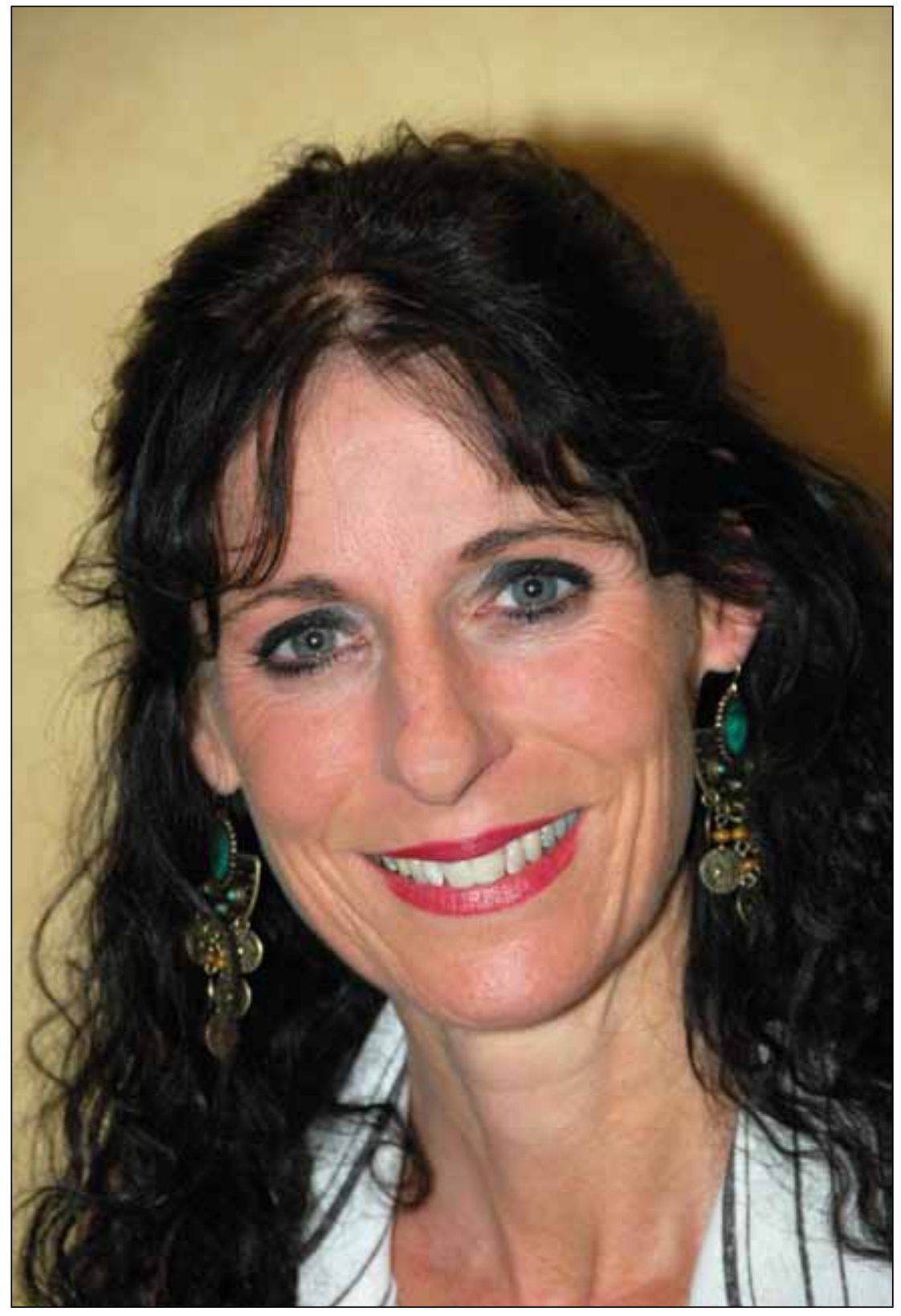

Heidi Kruger, Corporate Communications chief at the Board of Healthcare Funders (BHF).

The South African medical aid system has evolved in such a way (fragmented risk pools, legally beholden to prescribed minimum benefits (PMBs), a free-forall on provider tariffs), that there's little wonder it's adversarial and riven with antagonism, exploitation and mistrust.

These were some opinions expressed by funders and administrators in response to a KPMG medical scheme anti-fraud survey last month which found that code manipulation by service providers had increased by $51 \%$. This coincided with medical aid schemes reducing benefits and increasing premiums, significance, in spite of openly admitting that the system itself was 'highly conducive' to fraud. They explained that over the last nine years, covered by the tri-annual KPMG survey, their in-house forensic units had 'picked most of the low-hanging fruit' on the fraud activity tree, shifting their increasingly sophisticated detection knowledge to service providers. This, rather than any greater incidence of providers manipulating the system, accounted for the increase. The survey, compiled from figures supplied anonymously by the majority of the country's medical aid administrators, also found that member fraud (90\%) occurred overwhelmingly via non-disclosure of prior ailments, especially for high-cost conditions.

\section{They explained that over the last nine years, covered by the tri-annual KPMG survey, their in-house forensic units had 'picked most of the low- hanging fruit' on the fraud activity tree, shifting their increasingly sophisticated detection knowledge to service providers. This, rather than any greater incidence of providers manipulating the system, accounted for the increase.}

\section{Syndicates on the rise - $\mathrm{BHF}$}

Lynette Swanepoel, Manager of the Board of Healthcare Funders (BHF) Forensic Management Unit, revealed that some medical schemes no longer probed member non-disclosure 'fully or at all,' adding that her unit had noticed an upward trend in organised crime within the industry.

'It's safe to say that not all perpetrators of medical scheme fraud operate within a syndicated framework, but we do caution investigators and the general public to be aware that syndicated activities are the modus operandi in many instances, she added. She said coding irregularities were easy to detect, prove and recover, but muddied the waters when schemes were trying to establish disease profiles and determine new scheme benefits. Swanepoel appealed for the sharing of information about dodgy behaviour 'across silos' and for the lodging of joint industry complaints. Her unit pools claims data which enables a 'snapshot' of claiming patterns in a medical practice on any given day, and holds listings databases with vital information regarding perpetrators of 
fraud, including medical scheme members, employees and healthcare providers.

\section{'It's safe to say that not all perpetrators of medical} scheme fraud operate within a syndicated framework, but we do caution investigators and the general public to be aware that syndicated activities are the modus operandi in many instances.'

\section{Grudge purchase syndrome drives cynical behaviour}

Marius Smit, head of Discovery Health's Forensic Unit (Discovery is one of the 5\% of non-BHF members), agreed that unpacking coding-related fraud was relatively easy, but added that it 'will always be more difficult than probing those guys selling groceries and giving cash'.

'If you go back a decade or so, there was a lot of low hanging fruit in terms of dealing with healthcare fraud. The easier stuff got dealt with first. By the second [KPMG] survey the focus had moved to identifying the more complex type of frauds. The more resources you allocate to dealing with it, the more you uncover, he said. Smit added that because of the historic tensions, a healthcare provider's state of mind often predisposed them to 'bend the facts to get the most benefit from the medical scheme on behalf of their patient. Sometimes it's a legitimate error but sometimes a doctor (or any other service provider) will give us false information. He warned that medical aid schemes had a ready arsenal of punitive weapons, plus an obligation to report them to the Health Professions Council of South Africa (HPCSA), should they be found out. These included cancelling contractual agreements, paying members instead of providers and even changing the pre-authorisation process of patients. Smit agreed that common examples of provider/ patient collusion included using an incorrect code to enable hospital admission when a patient had only a hospital plan (versus more appropriately conducting the procedure in the doctor's rooms). A common provider fraud was 'code unbundling', plus claiming for 'things they never did without anyone ever being any the wiser - and it's even worse in the hospital environment; they bill a lot more than the usual doctor would do. Fraud was also common in cosmetic surgery, cosmetic dentistry and breast reduction surgery, where doctors attempted to label them as medically necessary when there was no supporting

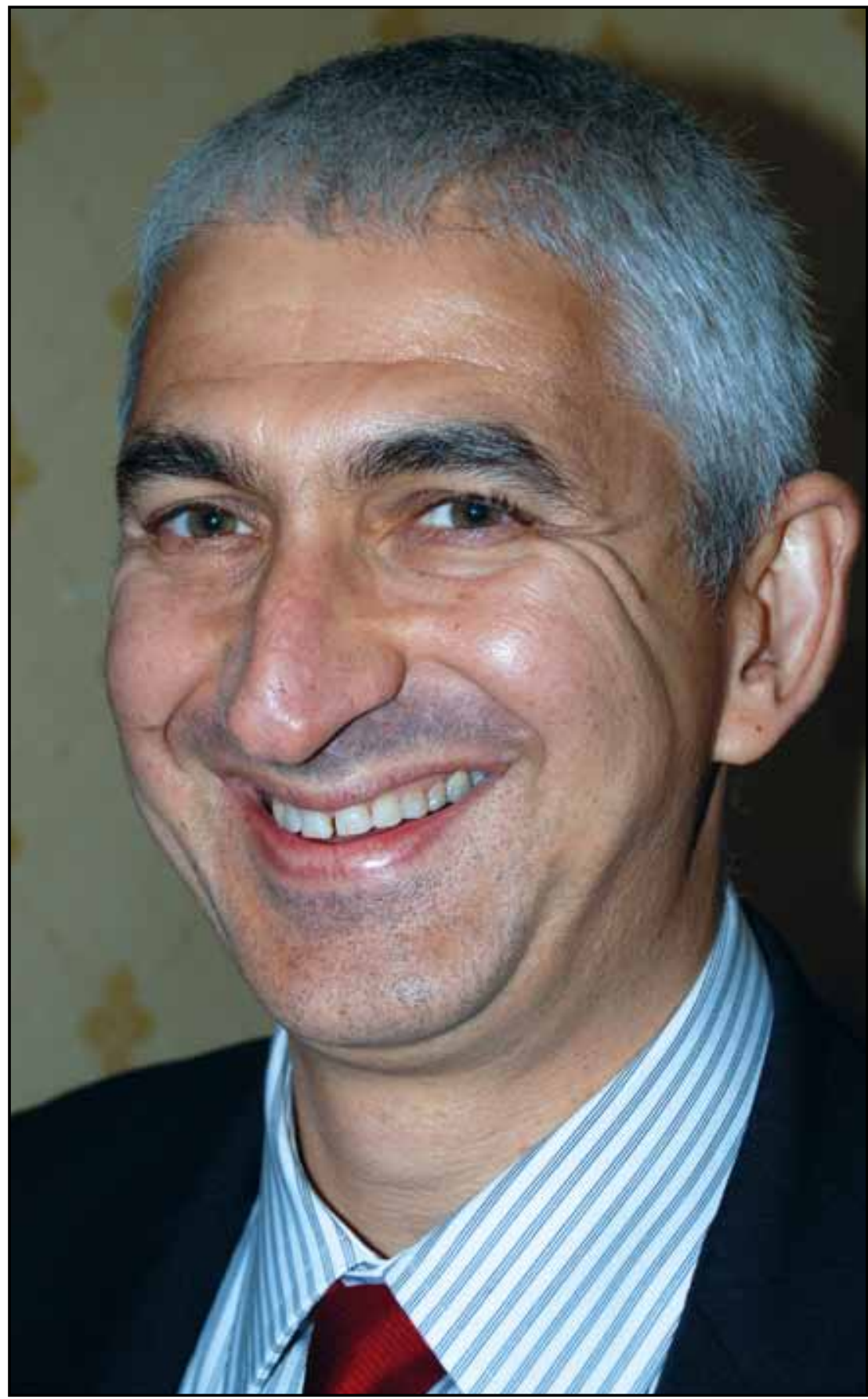

Dr Jonathan Broomberg, Discovery Health's CEO

clinical evidence. However, medicine was 'extremely dynamic' and exceptions arose. A recent example was bariatric bypass surgery (for extreme obesity), until now excluded, but with recent clinical evidence showing that 'in certain instances' it was medically indicated, with 'all sorts of benefits in terms of avoiding secondary problems'. Asked about regular media exposure of patients with rare and expensive (but excluded) conditions giving medical aid schemes 'negative press', Smit said his company had a vicarious responsibility to all members, not just individuals.

\section{Current maelstrom the antithesis of ubuntu}

Heidi Kruger, the BHF's Head of Corporate Communications, compared a medical scheme to a stokvel. 'I think it's safe to

This set-up is not conducive to cost containment and it's most certainly not about ubuntu, which it should

be. It's badly configured and providers don't necessarily think of health outcomes for the country. 


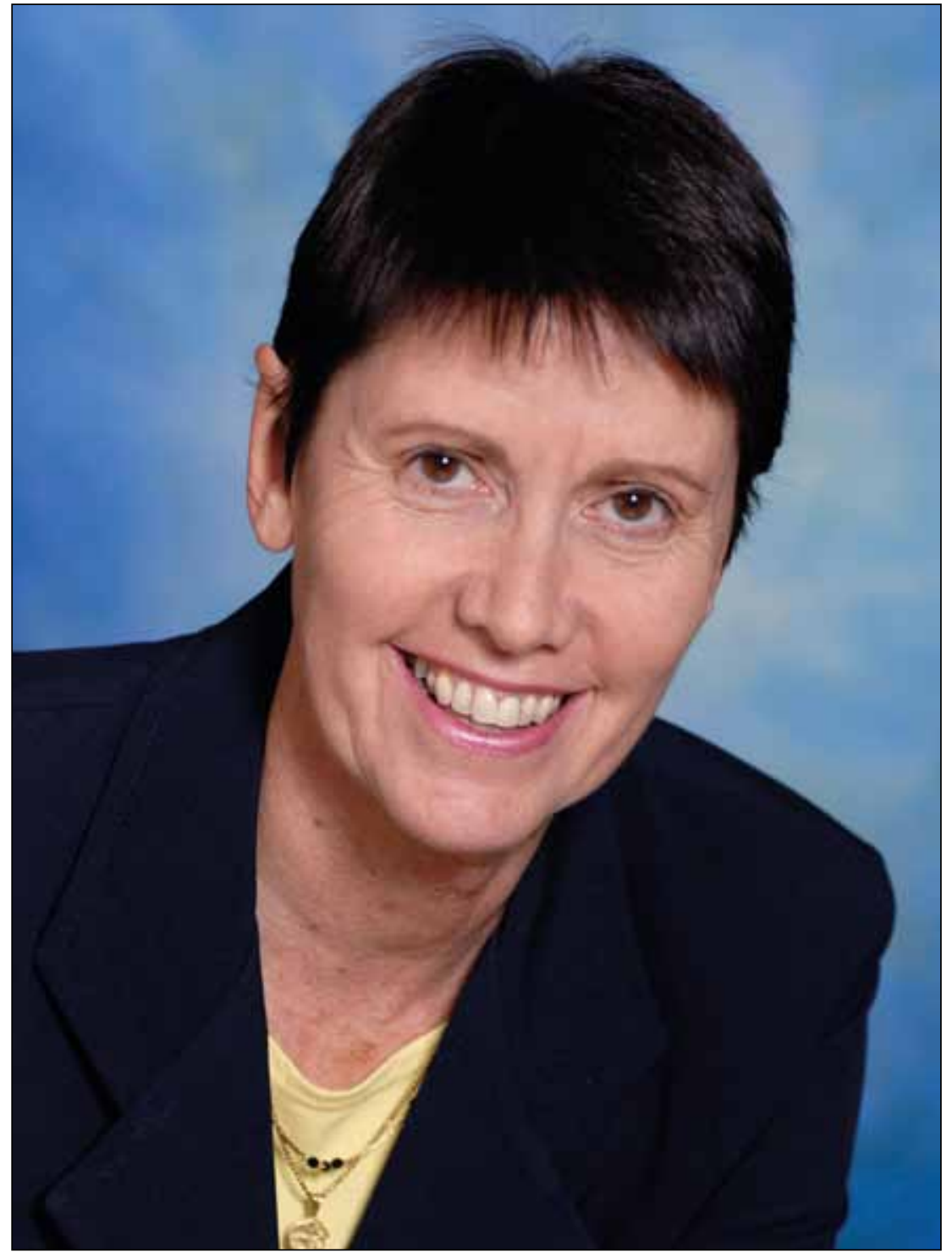

Camilla Singh, KPMG's National Forensic Director.

say that members often see a medical aid premium as a grudge purchase, so they'll get as much as possible out of it. They [and providers] don't necessarily understand that a medical scheme is just a pool of money and milking it contributes to the very premium increases they complain about - it's critical that an NHI or some other system comes into play. This setup is not conducive to cost containment and it's most certainly not about $u b u n t u$, which it should be. It's badly configured and providers don't necessarily think of health outcomes for the country. We need to get a patient well in the most efficient way possible and create a structure built on trust, with social solidarity. For example, the NHS in the UK is configured to give as many people as possible benefits in the most efficient manner. Here however fee-for-service means that the more the administrators of 'raking in profits while eroding benefits' and setting crippling annual ceilings on benefits.

\section{Schemes to take on DoH}

Medical schemes meanwhile are considering legal action against the national Department of Health following the derailment of plans to implement the department's proposed risk equalisation system that would standardise premiums and benefits across schemes. The Council for Medical Schemes (CMS) confessed that it was 'highly unlikely that a risk equalisation system would be implemented in the near future.

Discovery's CEO, Jonathan Broomberg, said finding the right balance between providing excellent, clinically appropriate benefits while keeping member contributions affordable on a sustainable basis was 'becoming increasingly difficult'. Ageing populations, more chronic diseases and cancer, plus costly new treatments and drugs pushed healthcare costs above the CPI. Therefore some schemes had failed to implement the price recommendations of the CMS. He said the internet petition interest group had based some of their statements on 'misleading information' which failed to accurately reflect Discovery's benefits or payments made to specific groups of healthcare providers.

Tembinkosi Bonakele, deputy commissioner of the Competition Commission, said a probe into healthcare costs could be on the cards. 'The issue has come up from various stakeholders - it concerns us and there seem to be limited tools to address it.'

\section{HPCSA acting registrar and CEO,}

Dr Kgosi Letlape, charged that people who spoke about patients as 'risk pools' were primarily concerned with making money and not about their clients' health.

HPCSA acting registrar and CEO, Dr Kgosi Letlape, charged that people who spoke about patients as 'risk pools' were primarily concerned with making money and not about their clients' health. 'How do human beings reduce themselves to the level where service to cars is better than the service to them?' he asked, adding that during his HPCSA tenure he intended addressing the Council's historic 'core negligence' of its legal obligation to set tariff guidelines. years, once-thriving dental practices going bankrupt, with dentists emigrating while the South African Medical Association accuses hospitals and scheme

\section{Chris Bateman}

chrisb@hmpg.co.za 